\title{
Approximate solution of the Bagley-Torvik equation by hybridizable discontinuous Galerkin methods
}

\author{
Mehmet Fatih Karaaslan, ${ }^{\mathrm{a}, *}$, Fatih Celiker ${ }^{\mathrm{b}, 1}$, Muhammet Kurulay ${ }^{\mathrm{c}, 2}$ \\ ${ }^{a}$ Department of Statistics, Yildiz Technical University, Istanbul, Turkey \\ ${ }^{b}$ Department of Mathematics, Wayne State University, Detroit, MI, USA. \\ ${ }^{c}$ Department of Mathematical Engineering, Yildiz Technical University, Istanbul, Turkey
}

\begin{abstract}
In this paper, we introduce a hybridizable discontinuous Galerkin method for numerically solving a boundary value problem associated with the BagleyTorvik equation that arises in the study of the motion of a plate immersed in a Newtonian fluid. One of the main features of these methods is that they are efficiently implementable since it is possible to eliminate all internal degrees of freedom and obtain a global linear system that only involves unknowns at the element interfaces. We display the results of a series of numerical experiments to ascertain the performance of the method.
\end{abstract}

Keywords: Hybridizable discontinuous Galerkin methods, Bagley-Torvik equation, fractional derivative, fractional calculus.

\section{Introduction}

Fractional calculus has received a considerable attention in the recent years. This is mainly due to the fact that they have found venues of applicability in many areas of science and engineering such as viscoelasticity,

\footnotetext{
*corresponding author

Email addresses: mfatih@yildiz.edu.tr (Mehmet Fatih Karaaslan), celiker@math. wayne.edu (Fatih Celiker), mkurulay@yildiz.edu.tr (Muhammet Kurulay)

${ }^{1}$ This author was partially supported by the National Science Foundation (Grant DMS1115280).

${ }^{2}$ This author was partially supported by the Office of Scientific Research Projects of Yildiz Technical University (Project no: 2013-01-03-DOP03).
} 
acoustics, electromagnetic waves, control theory, polarization, heat conduction, and mathematical biology, to name a few. A comprehensive literature review of fractional calculus and its applications can be found, for example, in $[20]$.

The Bagley-Torvik equation [2, 20] arises in modeling of the motion of a thin rigid plate immersed in a Newtonian fluid. The governing equation is given by the fractional differential equation

$$
A u^{\prime \prime}(x)+B D^{3 / 2} u(x)+C u(x)=f(x) \text { for } x \in \Omega=(0,1)
$$

subject to the initial conditions

$$
u(0)=u_{0}, \quad u^{\prime}(0)=u_{1} .
$$

Here, $u(x)$ represents the displacement of the plate of mass $M$ and surface area $S$. The constants $A, B$, and $C$ are given by

$$
A=M, \quad B=2 S \sqrt{\mu \rho}
$$

where $\mu$ and $\rho$ are the viscosity and density, respectively, of the fluid in which the plate is immersed, and

$$
C=K
$$

where $K$ is the stiffness of the spring to which the plate is attached. Finally, $f(x)$ represents the loading force. The operator $D^{\alpha}$, for $n-1<\alpha<n$, for some positive integer $n$, is the (left) Caputo fractional derivative which is defined as

$$
D^{\alpha} u(x)=\frac{1}{\Gamma(n-\alpha)} \int_{0}^{x}(x-t)^{n-\alpha-1} u^{(n)}(t) d t
$$

where $\Gamma(\cdot)$ is the gamma function defined by

$$
\Gamma(z)=\int_{0}^{\infty} e^{-t} t^{z-1} d t
$$

In this paper, we devise and implement HDG methods for a boundary value problem associated with the Bagley-Torvik equation (1.1)-(1.2). Our governing equation is a generalization of (1.1) to an arbitrary $\alpha$ with $1<\alpha<$ 2 in the fractional derivative term, namely, we take $n=2$ in (1.3). Hence,

$$
u^{\prime \prime}+D^{\alpha} u+u=f \quad \text { in } \Omega=(0,1)
$$


where we set $A=B=C=1$ merely for the clarity of the presentation, however, what we will present in the sequel can easily be generalized to arbitrary coefficients $A, B$, and $C$. We will consider solutions of (1.4) subject to the boundary conditions

$$
u=u_{D} \quad \text { on } \partial \Omega=\{0,1\} .
$$

where $u_{D}$ is a given function on the boundary of the domain $\Omega$. Similar problems have been considered in the literature by, for instance, by Al-Mdallal, Syam, Anwar [1], Ji and Tang [16], Rehman and Khan [23], and Staněk [24].

Next, we provide a brief overview of the recent efforts regarding the Bagley-Torvik equation and related problems. Our discussion is biased towards numerical methods devised for this purpose. Diethelm and Ford [14] solve the problem with Adams predictor and corrector method. Ray and Bera [21] employ an analytical approach to obtain the solution of the problem. Namely, they have used Adomian decomposition methods. Al-Mdallal, Syam, and Anwar [1] devise a numerical method for the solution of the Bagley-Torvik boundary value problem that is based on the combination of collocation and spline technique with shooting method. Çenesiz, Keskin and Kurnaz [10] suggest a new generalization of the Taylor collocation method. In [15], El-Sayed, Saleh, and Ziada solve a multi-term nonlinear fractional differential equation with Adomian decomposition method and another method that is called proposed numerical method. Li and Zhao [17] derive the Haar wavelet operational matrix of the fractional order integration and use it to solve the fractional order differential equations including the Bagley-Torvik, Ricatti, and composite fractional oscillation equations. In [25], Wang and Wang consider the Bagley-Torvik equation with 3/2-order fractional derivative and also a variation of it with $1 / 2$-order fractional derivative. They modify the equation into what they call a sequential fractional-order differential equation and consider certain properties of the analytical solution of the problem. Yüzbaşı [26] presents the Bessel collocation method to approximate the solution of the Bagley-Torvik equation. Mekkaoui and Hammouch [18] apply the fractional iteration method to general nonlinear differential equations of fractional order and implement their technique for the Bagley-Torvik equation. Ray [22] solves the equation by the Haar wavelet method. Ji and Tang [16] present high-order accurate Runge-Kutta local DG methods for boundary value problems involving fractional derivatives. Staněk [24] examines the existence of positive and negative solutions to a boundary value problem associated with the Bagley-Torvik equation. In [27], Yüzbaşı extends the 
Bessel collocation method to approximate solutions of the Volterra's population growth model and in [28] he employs a collocation method based on the Bernstein polynomials for fractional Riccati type differential equations. Most recently, Čermák and Kisela [11] studied the exact and discretized stability of the Bagley-Torvik equation and Mohammadi [19] devised a numerical method based on Chebyshev wavelet operational matrix of fractional derivative and applied the method to the Bagley-Torvik equation.

The HDG methods were introduced in [12] in the framework of second order elliptic problems. The main feature of these methods is that their approximate solutions can be expressed in an element-by-element fashion in terms of an approximate trace satisfying a global weak formulation. Therefore, they are easy to implement. For this and other features of HDG methods they have found numerous applications in the area of numerical methods for partial differential equations. Although there are too many of these application to list here we mention Cockburn and Mustapha [13] who introduced and analyzed HDG methods for a fractional partial differential equation. To the best of our knowledge this is the only paper that employs HDG methods for solving a fractional differential equation.

The rest of the paper is organized as follows. In Sec. 2, we introduce HDG methods for the problem under consideration. In Sec. 3, we state a characterization theorem that shows the efficiency of the HDG methods. In Sec. 4, we display a series of numerical experiments to ascertain the performance of the methods introduced. We end in Sec. 5 with some concluding remarks.

\section{HDG methods}

In this section, we define the HDG methods for the problem (1.4)-(1.5). We begin with defining an operator $\mathscr{L}$ by taking $n=2$ in (1.3) as follows

$$
D^{\alpha} u(x)=\frac{1}{\Gamma(2-\alpha)} \int_{0}^{x} u^{\prime \prime}(t)(x-t)^{1-\alpha} d t:=\mathscr{L}\left[u^{\prime \prime}(x)\right] .
$$

Then, by introducing two new unknowns, namely $p$ and $q$, we rewrite the governing equation (1.4) as the following system of equations 


$$
\begin{aligned}
q+u^{\prime}=0 & & \text { in } \Omega, \\
p+q^{\prime}=0 & & \text { in } \Omega, \\
p+\mathscr{L}[p]+u=f & & \text { in } \Omega .
\end{aligned}
$$

Given a positive integer $N$, we partition the domain $\Omega$ into $N$ elements by setting

$$
\Omega_{h}:=\left\{I_{j}=\left(x_{j-1}, x_{j}\right): 0=x_{0}<x_{1}<\cdots<x_{N-1}<x_{N}=1\right\} .
$$

We associate the set of nodes, $\mathscr{E}_{h}:=\left\{x_{0}, x_{1}, \ldots, x_{N}\right\}$, and the set of interior nodes $\mathscr{E}_{h}^{\circ}:=\mathscr{E}_{h} \backslash \partial \Omega$; we also set $\partial \Omega_{h}:=\left\{\partial K: K \in \Omega_{h}\right\}$. For each element $K \in \Omega_{h}$, let $h_{K}$ denote the length of $K$, and set $h:=\max _{K \in \Omega_{h}}\left\{h_{K}\right\}$. Finally, for any given polynomial degree $k \geq 0$ and an element $K \in \Omega_{h}$, we define $\mathcal{P}^{k}(K)$ as the set of polynomials of degree less than or equal to $k$ on $K$. The space of piecewise polynomials of degree $k$ on $\Omega$ is defined accordingly as

$$
V_{h}^{k}:=\left\{v: \Omega_{h} \mapsto \mathbb{R}:\left.v\right|_{K} \in \mathcal{P}^{k}(K) \text { for all } K \in \Omega_{h}\right\}
$$

We also set

$$
L_{0}^{2}\left(\mathscr{E}_{h}\right):=\left\{m \in L^{2}\left(\mathscr{E}_{h}\right): m=0 \text { on } \partial \Omega\right\}
$$

where $L^{2}\left(\mathscr{E}_{h}\right)$ is simply a copy of the Euclidean space $\mathbb{R}^{N+1}$.

The HDG methods seek an approximation $\left(q_{h}, p_{h}, u_{h}, \widehat{u}_{h}\right)$ to the exact solution $\left(q, p, u,\left.u\right|_{\mathscr{E}_{h}}\right)$, of $(2.1)$, in the finite dimensional space $V_{h}^{k} \times V_{h}^{k} \times$ $V_{h}^{k} \times L^{2}\left(\mathscr{E}_{h}\right)$. It is determined by requiring that

$$
\begin{aligned}
& \left(q_{h}, v\right)-\left(u_{h}, v^{\prime}\right)+\left\langle\widehat{u}_{h}, v n\right\rangle=0, \\
& \left(p_{h}, w\right)-\left(q_{h}, w^{\prime}\right)+\left\langle\widehat{q}_{h}, w n\right\rangle=0, \\
& \left(p_{h}, z\right)+\left(\mathscr{L}\left[p_{h}\right], z\right)+\left(u_{h}, z\right)=(f, z), \\
& \left\langle\widehat{q}_{h} n, \mu\right\rangle=0 \text {, }
\end{aligned}
$$

hold for all

$$
(v, w, z, \mu) \in V_{h}^{k} \times V_{h}^{k} \times V_{h}^{k} \times L_{0}^{2}\left(\mathscr{E}_{h}\right) .
$$

Here, the outward unit normal vectors are $n\left(x^{\mp}\right):= \pm 1$ for $x \in \mathscr{E}_{h}$. The "volume" inner product is defined as

$$
(u, v):=\sum_{K \in \Omega_{h}}(u, v)_{K} \quad \text { where } \quad(u, v)_{K}:=\int_{K} u(x) v(x) d x
$$


and the boundary inner product is defined as $\langle u, v n\rangle:=\sum_{K \in \Omega_{h}}\langle u, v n\rangle_{\partial K} \quad$ where $\quad\langle u, v\rangle_{\partial K}:=u\left(x_{j}^{-}\right) v\left(x_{j}^{-}\right)+u\left(x_{j-1}^{+}\right) v\left(x_{j-1}^{+}\right)$,

when $K=\left(x_{j-1}, x_{j}\right)$, and $u\left(x^{ \pm}\right):=\lim _{\epsilon \downarrow} u(x \pm \epsilon)$ for $x \in \mathscr{E}_{h}$. The boundary condition (1.5) is imposed by requiring that

$$
\widehat{u}_{h}=u_{D} \quad \text { on } \partial \Omega \text {. }
$$

The numerical trace $\widehat{q}_{h}$ is defined as

$$
\widehat{q}_{h}=q_{h}+\tau\left(u_{h}-\widehat{u}_{h}\right) n
$$

where $\tau$ is a nonnegative function defined on $\partial \Omega_{h}$. This completes the definition of the HDG method.

\section{Characterization of the Approximate Solution}

In this section, we show that the only globally coupled unknowns of the HDG method defined by the weak formulation (2.2), and the formula (2.3) for the numerical traces are the approximations at the nodes $\widehat{u}_{h}$. We also show that the remaining components of the approximate solution can be expressed solely in terms of element-by-element-defined operators acting on $\widehat{u}_{h}$. To do this, we follow the framework provided in [12], [5], and [7].

We begin with introducing the above-mentioned locally defined operators which we call the local solvers associated with the method. The first local solver is defined on the element $K \in \Omega_{h}$ as the mapping

$$
\mu \in L^{2}(\partial K) \mapsto(\mathrm{Q} \mu, \mathrm{P} \mu, \mathrm{U} \mu) \in\left[\mathcal{P}^{k}(K)\right]^{3}
$$

where

$$
\begin{array}{llll}
(\mathrm{Q} \mu, v)_{K} & -\left(\mathrm{U} \mu, v^{\prime}\right)_{K} & & =-\langle\mu, v n\rangle_{\partial K}, \\
(\mathrm{P} \mu, w)_{K} & -\left(\mathrm{Q} \mu, w^{\prime}\right)_{K} & +\langle\widehat{\mathrm{Q}} \mu, w n\rangle_{\partial K} & =0 \\
(\mathrm{P} \mu, z)_{K} & +(\mathscr{L}[\mathrm{P} \mu], z)_{K} & +(\mathrm{U} \mu, z)_{K} & =0
\end{array}
$$

for all $v, w, z \in \mathcal{P}^{k}(K)$ where

$$
\widehat{\mathrm{Q}} \mu=\mathrm{Q} \mu+\tau(\mathrm{U} \mu-\mu) n \quad \text { on } \partial K .
$$


The second local solver is defined on the element $K \in \Omega_{h}$ as the mapping

$$
f \in L^{2}(\partial K) \mapsto(\mathrm{Q} f, \mathrm{P} f, \mathrm{U} f) \in\left[\mathcal{P}^{k}(K)\right]^{3}
$$

where

$$
\begin{array}{llll}
(\mathrm{Q} f, v)_{K} & -\left(\mathrm{U} f, v^{\prime}\right)_{K} & & =0, \\
(\mathrm{P} f, w)_{K} & -\left(\mathrm{Q} f, w^{\prime}\right)_{K} & +\langle\widehat{\mathrm{Q}} f, w n\rangle_{\partial K} & =0, \\
(\mathrm{P} f, z)_{K} & +(\mathscr{L}[\mathrm{P} f], z)_{K} & +(\mathrm{U} f, z)_{K} & =(f, z)_{K},
\end{array}
$$

for all $v, w, z \in \mathcal{P}^{k}(K)$ where

$$
\widehat{\mathrm{Q}} f=\mathrm{Q} f+\tau(\mathrm{U} f) n \quad \text { on } \partial K .
$$

Observe that (3.1) defines an approximation $(\mathrm{Q} \mu, \mathrm{P} \mu, \mathrm{U} \mu)$ to the solution $(q, p, u)$ of the problem

$$
\begin{aligned}
q+u^{\prime}=0 & \text { in } K, \\
p+q^{\prime}=0 & \text { in } K, \\
p+\mathscr{L}[p]+u=0 & \text { in } K,
\end{aligned}
$$

subject to the boundary conditions

$$
u=\mu \quad \text { on } \partial K,
$$

whereas (3.2) defines an approximation $(\mathrm{Q} f, \mathrm{P} f, \mathrm{U} f)$ to the solution $(q, p, u)$ of the problem

$$
\begin{aligned}
q+u^{\prime}=0 & \text { in } K, \\
p+q^{\prime}=0 & \text { in } K, \\
p+\mathscr{L}[p]+u=f & \text { in } K,
\end{aligned}
$$

subject to the boundary conditions

$$
u=0 \quad \text { on } \partial K .
$$

Let us note that the solution of the problems (3.1) and (3.2) require the solution of a linear system of size $(k+1) \times(k+1)$ and hence it is extremely easy. Furthermore, since the definition is local in the sense that (3.1) and (3.2) are defined on a single element $K \in \Omega_{h}$, the computation is trivially parallelizable. 
The function $u_{D}$, as well as any other function defined only on $\partial \Omega$ is extended to $\mathscr{E}_{h}$ by zero. We also set

$$
\lambda_{h}:= \begin{cases}\widehat{u}_{h} & \text { on } \partial \Omega_{h} \backslash \partial \Omega \\ 0 & \text { on } \partial \Omega,\end{cases}
$$

so that we have that $\widehat{u}_{h}=\lambda_{h}+u_{D}$ where $\lambda_{h} \in L_{0}^{2}\left(\mathscr{E}_{h}\right)$. We can now state a theorem that characterizes the HDG approximate solution in terms of the local solvers.

Theorem 3.1. The HDG solution $\left(q_{h}, p_{h}, u_{h}, \lambda_{h}\right) \in\left[V_{h}^{k}\right]^{3} \times L_{0}^{2}\left(\mathscr{E}_{h}\right)$ given by the HDG method (2.2) can be expressed in terms of the local solvers as

$$
\begin{aligned}
& q_{h}=\mathrm{Q} \lambda_{h}+\mathrm{Q} u_{D}+\mathrm{Q} f, \\
& p_{h}=\mathrm{P} \lambda_{h}+\mathrm{P} u_{D}+\mathrm{P} f, \\
& u_{h}=\mathrm{U} \lambda_{h}+\mathrm{U} u_{D}+\mathrm{U} f,
\end{aligned}
$$

where $\lambda_{h}$ satisfies

$$
a_{h}\left(\lambda_{h}, \mu\right)=b_{h}(\mu), \quad \text { for all } \mu \in L_{0}^{2}\left(\mathscr{E}_{h}\right),
$$

where we have defined

$$
\begin{aligned}
a_{h}\left(\lambda_{h}, \mu\right) & =-\left\langle\widehat{\mathrm{Q}} \lambda_{h}, \mu n\right\rangle_{\partial \Omega_{h}}, \\
b_{h}(\mu) & =\left\langle\widehat{\mathrm{Q}} u_{D}+\widehat{\mathrm{Q}} f, \mu n\right\rangle_{\partial \Omega_{h}} .
\end{aligned}
$$

The proof of this theorem is straightforward since (3.3) is nothing but a rewriting of the last equation (namely, the conservativity condition) in (2.2). For details we refer to $[12,5,7]$.

Note that the total number of globally coupled unknowns in the equation in (3.3) is $N-1$ where $N$ is the number of elements in $\Omega_{h}$. In particular, it is independent of the polynomial degree $k$. This should be contrasted with the total number of globally coupled unknowns in the original form of the equation (2.2) that defines the HDG method, namely, $3 N(k+1)+N-1$. Thus, the result in Theorem 3.1 shows that the total number of globally coupled unknowns can be significantly reduced. This is what we mean when we say that HDG methods are efficiently implementable. 


\section{Numerical Examples}

In this section, we display the results of some numerical experiments to assess the performance of the HDG methods described. In all of the examples we compute an approximation to the solution of the problem using the method (2.2) where we take $\tau=1 / h$ in (2.3) in the definition of the numerical trace $\widehat{q}_{h}$. We employ the HDG method with $k=1,2$, and 3 . Although the method is well defined and convergent for $k=0$, we observed that it converges to an incorrect solution. This requires further investigation and that's why we do not report results for $k=0$ in this paper.

In Tables $1-5$, for $\varphi=u, q, p,\left\|\varphi-\varphi_{h}\right\|_{0, \Omega}$ denotes the $L^{2}$-error computed by

$$
\left\|\varphi-\varphi_{h}\right\|_{0, \Omega}=\left(\sum_{K \in \Omega_{h}} \int_{K}\left(\varphi-\varphi_{h}\right)^{2}\right)^{\frac{1}{2}} .
$$

We display these quantities in the "error" column in our tables. The approximate convergence order of the error is then displayed in the "order" column next to the error column, and it contains the logarithm of the ratio of the two consecutive errors corresponding to two HDG solutions with two consecutive meshes. Therefore, for instance, if the numbers in the order column approaches $\kappa$, it indicates that the error satisfies a formula of the form

$$
\left\|\varphi-\varphi_{h}\right\|_{0, \Omega} \leq C h^{\kappa}
$$

for some constant $C$ that is independent of the mesh size $h$, that is, the method converges with order $\kappa$ with respect to $h$. In the last two columns of Tables 1 and 2, we carry out a similar study for the error in the numerical trace $\widehat{u}_{h}$. Therein, in the error column we display the $\ell^{\infty}$-error

$$
\left\|u-\widehat{u}_{h}\right\|_{\infty, \mathscr{E}_{h}}=\max _{e \in \mathscr{E}_{h}}\left|\left(u-\widehat{u}_{h}\right)(e)\right| .
$$

In our first example, we consider the problem

$$
\begin{aligned}
& u^{\prime \prime}(x)+D^{3 / 2} u(x)+u(x)=2+4 \sqrt{x / \pi}+x^{2} \quad \text { for } x \in \Omega, \\
& u(0)=0, \quad u(1)=1 .
\end{aligned}
$$

The exact solution of this problem is given by $u(x)=x^{2}$ since $D^{3 / 2}\left(x^{2}\right)=$ $4 \sqrt{x / \pi}$. We display our results in Table 1 . We observe that for $k=1, u_{h}$ and $p_{h}$ both converge with the optimal order $k+1=2$ whereas $q_{h}$ converges 
with the suboptimal order $k=1$. We also see that the numerical trace $\widehat{u}_{h}$ also converges with order $k+1=2$. Although this is remarkable, we do not see a superconvergence phenomenon at the nodes of the mesh. This should be contrasted with the behavior of the DG and HDG methods for problems in one space dimension for other boundary value problems that involve only the ordinary derivative. For such problems the numerical trace typically superconverges with order $2 k+1$. For a detailed discussion of this and related matters we refer the reader to $[3,4,5,6,7,8,9]$. The behavior of the error $\left\|u-\widehat{u}_{h}\right\|_{\infty, \mathscr{E}_{h}}$ in Table 1 shows that the numerical traces of the HDG method for fractional boundary value problems do not have such a superconvergence property. Although this is a negative result, it is worth noting it here.

For $k=2$, what we see from Table 1 is that the HDG method captures the exact solution (up to the machine accuracy). This is quite typical of DG and HDG methods, namely, whenever the exact solution happens to be a member of the finite element space, the DG and HDG methods, no matter how coarse the mesh is, capture that solution. This is the reason why we see errors that are practically zero, and that's why we do not display the numbers in the "order" column for this example. For a proof of this phenomenon for DG and HDG methods in the context of the boundary value problems with integer order derivatives we refer to $[3,4,8]$.

In our second example, we consider the equation

$$
\begin{aligned}
& u^{\prime \prime}(x)+D^{\alpha} u(x)+u(x)=\frac{\Gamma(4)}{\Gamma(4-\alpha)} x^{3-\alpha}+x^{3}+7 x+1 \quad \text { for } x \in \Omega, \\
& u(0)=1, \quad u(1)=3 .
\end{aligned}
$$

where $1<\alpha<2$ is an arbitrary parameter. For any such $\alpha$ the exact solution is given by $u(x)=x^{3}+x+1$. In Table 2 , we display the history of convergence for $\alpha=3 / 2$. We observe that when $k=1$, the convergence for $u_{h}$ and $p_{h}$ is of the optimal order $k+1=2$ and $q_{h}$ converges with the suboptimal order $k=1$. Similarly, $\widehat{u}_{h}$ converges with order $k+1=2$. These results are in agreement with what we observed in Table 1 . For $k=2, u_{h}$ still converges with the optimal order $k+1=3$ but this time both $p_{h}$ and $q_{h}$ converge suboptimally with order $k=2$. The numerical trace $\widehat{u}_{h}$ also converges suboptimally with order $k=2$. Since the exact solution is a polynomial of degree 3 , for $k=3$ the HDG solution coincides with the exact solution and hence the errors are zero up to the machine accuracy. This is what we observe in the last block row $(k=3)$ of Table 2 . 
Finally, in Tables 3-5 we carry out a study of the convergence behavior of the HDG method for various values of the fractional derivative parameter $\alpha$. We display $\left\|u-u_{h}\right\|_{0, \Omega}$ for $\alpha=1.1,1.2,1.5,1.7$, and 1.9. We start with a mesh with $N=4$ elements and refine it up to a mesh with $N=128$ elements. We do not display the results for the $N=4$ mesh since we can start displaying the order column only after the first refinement of the mesh. In Table 3, we employ the HDG method to solve the problem (4.2) with polynomials of degree $k=1$. We see that for all values of the parameter $\alpha$, the method converges with the optimal order $k+1=2$. In Table 4 we display results for $k=2$, and we again see that the method converges with the optimal order $k+1=3$ for all values of $\alpha$. In Table 5 we display results for $k=3$. Since the exact solution is a third order polynomial regardless of the value of $\alpha$, the HDG method is expected to capture the exact solution. Our results in Table 5 are in agreement with this. Finally, let us note that we observed (for various values of $\alpha$ ) similar behavior of convergence for $\left\|p-p_{h}\right\|_{0, \Omega},\left\|q-q_{h}\right\|_{0, \Omega},\left\|u-\widehat{u}_{h}\right\|_{\infty, \mathscr{E}_{h}}$ as we have observed in Table 1 and Table 2 for $\alpha=3 / 2$. However, we omit displaying these results herein. This indicates that the HDG methods are robust with respect to the order of the fractional derivative.

\section{Conclusion}

We have introduced HDG methods for a boundary value problem associated with the Bagley-Torvik equation that involves fractional order derivatives as well as a second order derivative term. We showed that these methods are efficiently implementable since the only globally coupled unknowns are those associated with the numerical trace $\widehat{u}_{h}$ of the primal variable $u_{h}$. The interior degrees freedom for $u_{h}$ and the remaining variables can then be recovered from $\widehat{u}_{h}$ by an element-by-element computation. We have displayed numerical results to ascertain the convergence behavior of the method and we observed that when polynomials of degree at most $k$ are used for all unknowns, $u_{h}$ converges with the optimal order $k+1, p_{h}$ and $q_{h}$ seem to converge slightly suboptimally with order $k$. Obtaining proofs of these convergence results constitutes the subject of ongoing work. We are working on devising HDG methods for other problems with fractional derivatives. 


\section{References}

[1] Q. M. Al-Mdallal, M. I. Syam, and M.N. Anwar, A collocation-shooting method for solving fractional boundary value problems, Comm. in Nonlinear Sci. and Numer. Sim. 15 (2010), no. 12, 3814-3822.

[2] R.L. Bagley and P. J.Torvik, On the appearance of the fractional derivative in the behavior of real materials, ASME Trans. J. Appl. Mech. 51 (1984), 294-298.

[3] F. Celiker and B. Cockburn, Element-by-element post-processing of discontinuous Galerkin methods for Timoshenko beams, J. Sci. Comput. 27 (2006), no. 1-3, 177-187.

[4] F. Celiker and B. Cockburn, Superconvergence of the numerical traces of discontinuous Galerkin and hybridized methods for convection-diffusion problems in one space dimension, Math. Comp. 76 (2007), no. 257, 6796.

[5] F. Celiker, B. Cockburn, and K. Shi, Hybridizable discontinuous Galerkin methods for Timoshenko beams, J. Sci. Comput. 44 (2010), $1-37$.

[6] F. Celiker, B. Cockburn, and K. Shi, A projection-based error analysis of HDG methods for Timoshenko beams, Math. Comp. 81 (2012), 131-151.

[7] F. Celiker and L. Fan, HDG methods for Naghdi arches, JSC 59 (2014), $217-246$.

[8] F. Celiker, L. Fan, S. Zhang, and Z. Zhang, Locking-free optimal discontinuous Galerkin methods for a Naghdi-type arch model, J. Sci. Comput. 52 (2012), 49-84.

[9] F. Celiker, L. Fan, and Z. Zhang, Element-by-element post-processing of DG methods for Naghdi arches., Int. J. Numer. Anal. Model. 8 (2011), no. 3, 391-409.

[10] Y. Çenesiz, Y. Keskin, and A. Kurnaz, The solution of the Bagley-Torvik equation with the generalized Taylor collocation method, J. Franklin Institute 347 (2010), 452-466. 
[11] J. Čermák and T. Kisela, Exact and discretized stability of the BagleyTorvik equations, J. Comput. Appl. Math. 269 (2014), 53-67.

[12] B. Cockburn, J. Gopalakrishnan, and R. Lazarov, Unified hybridization of discontinuous Galerkin, mixed, and continuous Galerkin methods for second order elliptic problems, SIAM J. Numer. Anal. 47 (2009), no. 2, 1319-1365.

[13] B. Cockburn and K. Mustapha, A hybridizable discontinuous Galerkin method for fractional diffusion problems, Numer. Math. 130 (2015), no. 2, 293-314.

[14] K. Diethelm and N. J. Ford, Numerical solution of the Bagley-Torvik equation, BIT Numerical Mathematics 42 (2002), no. 3, 490-507.

[15] A.M.A. El-Sayed, M.M. Saleh, and E.A.A. Ziada, Analytical and numerical solutions of multi-term nonlinear fractional orders differential equations, Appl. Numer. Math. 60 (2010), no. 8, 788-797.

[16] X. Ji and H. Tang, High-order accurate Runge-Kutta (local) discontinuous Galerkin methods for one- and two- dimensional fractional diffusion equations, Numer. Math. Theor. Meth. Appl. 5 (2012), no. 3, 333-358.

[17] Y. Li and W. Zhao, Haar wavelet operational matrix of fractional order integration and its applications in solving the fractional order differential equations, Applied Mathematics and Computation 216 (2010), no. 8, 2276-2285.

[18] T. Mekkaoui and Z. Hammouch, Approximate analytical solutions to the Bagley-Torvik equation by the fractional iteration method, Annals of the University of Craiova Mathematics and Computer Science Series. 39 (2012), no. 2, 251-256.

[19] F. Mohammadi, Numerical solution of Bagley-Torvik equation using Chebyshev wavelet operational matrix of fractional derivative, Int. J. Adv. in Appl. Math. and Mech. 2 (2014), no. 1, 83-91.

[20] I. Podlubny, Fractional differential equations, Academic Press, San Diego, 1999. 
[21] S. S. Ray and R. K. Bera, Analytical solution of the Bagley-Torvik equation by Adomian decomposition method, Applied Mathematics and Computation 168 (2005), no. 1, 398-410.

[22] S.S. Ray, On Haar wavelet operational matrix of general order and its application for the numerical solution of fractional Bagley-Torvik equation, Applied Mathematics and Computation 218 (2012), 5239-5248.

[23] M. Rehman and R. Khan, A numerical method for solving boundary value problems for fractional differential equations, Appl. Math. Modelling 36 (2012), 894-907.

[24] S. Staněk, Two-point boundary value problems for the generalized Bagley-Torvik fractional differential equation, Cent. Eur. J. Math. 11 (2013), 574-593.

[25] Z.H. Wang and X. Wang, General solution of the Bagley-Torvik equation with fractional-order derivative, Comm. in Nonlinear Sci. and Numer. Sim. 15 (2010), no. 5, 1279-1285.

[26] Ş. Yüzbaşı, Numerical solution of the Bagley-Torvik equation by the Bessel collocation method, 36 (2012), no. 3, 300-312.

[27] Ş. Yüzbaş1, A numerical approximation for Volterra's population growth model with fractional order, Applied Mathematical Modelling 37 (2013), no. $5,3216-3227$.

[28] Ş. Yüzbaşı, Numerical solutions of fractional Riccati type differential equations by means of the Bernstein polynomials, Applied Mathematics and Computation 219 (2013), no. 11, 6328-6343. 

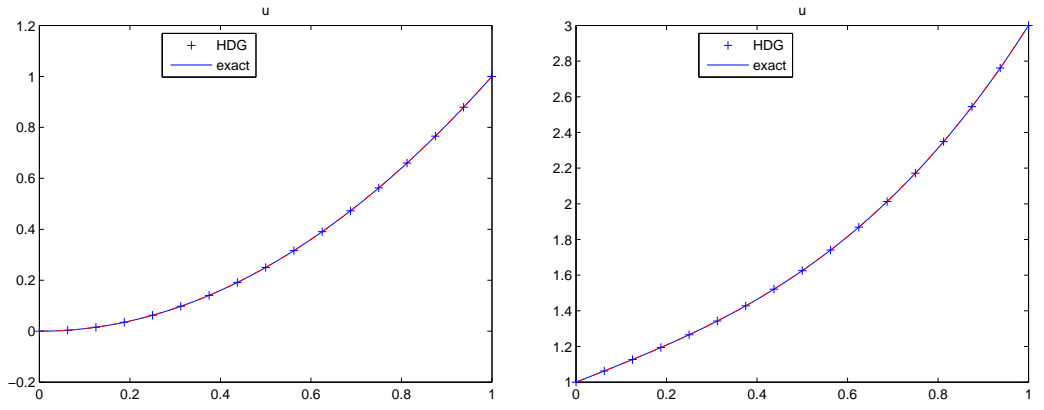

Figure 1: HDG and exact solutions for problem (4.1) (left) and (4.2) (right) with $\alpha=1.5$, $k=1$, and $N=16$.

Table 1: History of convergence for the problem (4.1).

\begin{tabular}{cccccccccc}
\hline \hline & & \multicolumn{2}{c}{$\left\|u-u_{h}\right\|_{0, \Omega}$} & \multicolumn{2}{c}{$\left\|q-q_{h}\right\|_{0, \Omega}$} & \multicolumn{2}{c}{$\left\|p-p_{h}\right\|_{0, \Omega}$} & \multicolumn{2}{c}{$\left\|u-\widehat{u}_{h}\right\|_{L^{2}\left(\mathscr{E}_{h}\right)}$} \\
\hline$k$ & $N$ & error & order & error & order & error & order & error & order \\
\hline & 16 & $3.07 \mathrm{e}-04$ & 2.00 & $5.16 \mathrm{e}-03$ & 1.00 & $5.56 \mathrm{e}-05$ & 2.00 & $6.70 \mathrm{e}-06$ & 2.00 \\
1 & 32 & $7.68 \mathrm{e}-05$ & 2.00 & $2.58 \mathrm{e}-03$ & 1.00 & $1.39 \mathrm{e}-05$ & 2.00 & $1.68 \mathrm{e}-06$ & 2.00 \\
& 64 & $1.92 \mathrm{e}-05$ & 2.00 & $1.29 \mathrm{e}-03$ & 1.00 & $3.48 \mathrm{e}-06$ & 2.00 & $4.20 \mathrm{e}-07$ & 2.00 \\
& 128 & $4.80 \mathrm{e}-06$ & 2.00 & $6.44 \mathrm{e}-04$ & 1.00 & $8.69 \mathrm{e}-07$ & 2.00 & $1.05 \mathrm{e}-07$ & 2.00 \\
\hline & 4 & $4.67 \mathrm{e}-16$ & - & $1.19 \mathrm{e}-15$ & - & $4.13 \mathrm{e}-15$ & - & $4.16 \mathrm{e}-16$ & - \\
2 & 8 & $3.83 \mathrm{e}-15$ & - & $1.29 \mathrm{e}-14$ & - & $1.15 \mathrm{e}-13$ & - & $5.11 \mathrm{e}-15$ & - \\
& 16 & $2.00 \mathrm{e}-14$ & - & $6.97 \mathrm{e}-14$ & - & $4.30 \mathrm{e}-13$ & - & $2.75 \mathrm{e}-14$ & - \\
& 32 & $3.64 \mathrm{e}-14$ & - & $1.28 \mathrm{e}-13$ & - & $1.57 \mathrm{e}-12$ & - & $5.28 \mathrm{e}-14$ & - \\
\hline \hline
\end{tabular}


Table 2: History of convergence for problem (4.2) with $\alpha=1.5$.

\begin{tabular}{cccccccccc}
\hline \hline & \multicolumn{1}{c}{$\left\|u-u_{h}\right\|_{0, \Omega}$} & \multicolumn{2}{c}{$\left\|q-q_{h}\right\|_{0, \Omega}$} & \multicolumn{2}{c}{$\left\|p-p_{h}\right\|_{0, \Omega}$} & \multicolumn{2}{c}{$\left\|u-\widehat{u}_{h}\right\|_{L^{2}\left(\mathscr{E}_{h}\right)}$} \\
\hline$k$ & $N$ & error & order & error & order & error & order & error & order \\
\hline & 16 & $5.30 \mathrm{e}-04$ & 2.03 & $8.96 \mathrm{e}-03$ & 1.02 & $1.43 \mathrm{e}-04$ & 2.19 & $1.45 \mathrm{e}-05$ & 2.00 \\
& 32 & $1.32 \mathrm{e}-04$ & 2.01 & $4.47 \mathrm{e}-03$ & 1.00 & $3.44 \mathrm{e}-05$ & 2.06 & $3.63 \mathrm{e}-06$ & 2.00 \\
& 64 & $3.30 \mathrm{e}-05$ & 2.00 & $2.23 \mathrm{e}-03$ & 1.00 & $8.52 \mathrm{e}-06$ & 2.01 & $9.08 \mathrm{e}-07$ & 2.00 \\
& 128 & $8.23 \mathrm{e}-06$ & 2.00 & $1.12 \mathrm{e}-03$ & 1.00 & $2.13 \mathrm{e}-06$ & 2.00 & $2.27 \mathrm{e}-07$ & 2.00 \\
\hline & 8 & $3.73 \mathrm{e}-05$ & 3.00 & $3.18 \mathrm{e}-04$ & 2.00 & $1.04 \mathrm{e}-05$ & 2.21 & $1.08 \mathrm{e}-06$ & 1.97 \\
2 & 16 & $4.67 \mathrm{e}-06$ & 3.00 & $7.94 \mathrm{e}-05$ & 2.00 & $2.49 \mathrm{e}-06$ & 2.06 & $2.73 \mathrm{e}-07$ & 1.99 \\
& 32 & $5.84 \mathrm{e}-07$ & 3.00 & $1.99 \mathrm{e}-05$ & 2.00 & $6.16 \mathrm{e}-07$ & 2.02 & $6.83 \mathrm{e}-08$ & 2.00 \\
& 64 & $7.38 \mathrm{e}-08$ & 2.99 & $4.96 \mathrm{e}-06$ & 2.00 & $1.54 \mathrm{e}-07$ & 2.00 & $1.71 \mathrm{e}-08$ & 2.00 \\
\hline \multirow{3}{*}{3} & 4 & $1.43 \mathrm{e}-13$ & - & $5.08 \mathrm{e}-13$ & - & $2.55 \mathrm{e}-12$ & - & $1.98 \mathrm{e}-13$ & - \\
& 8 & $6.06 \mathrm{e}-13$ & - & $2.28 \mathrm{e}-12$ & - & $2.04 \mathrm{e}-11$ & - & $8.99 \mathrm{e}-13$ & - \\
& 16 & $4.67 \mathrm{e}-12$ & - & $1.61 \mathrm{e}-11$ & - & $8.96 \mathrm{e}-11$ & - & $6.81 \mathrm{e}-12$ & - \\
\hline \hline
\end{tabular}

Table 3: $\left\|u-u_{h}\right\|_{0, \Omega}$ for problem (4.2) with $k=1$.

\begin{tabular}{c|cccccccccc}
\hline \hline$N$ & \multicolumn{2}{|c}{8} & \multicolumn{2}{c}{16} & \multicolumn{2}{c}{32} & \multicolumn{2}{c}{64} & \multicolumn{2}{c}{128} \\
\hline$\alpha$ & error & order & error & order & error & order & error & order & error & order \\
\hline 1.9 & $2.18 \mathrm{e}-03$ & 2.10 & $5.34 \mathrm{e}-04$ & 2.03 & $1.33 \mathrm{e}-04$ & 2.01 & $3.31 \mathrm{e}-05$ & 2.00 & $8.28 \mathrm{e}-06$ & 2.00 \\
1.7 & $2.17 \mathrm{e}-03$ & 2.10 & $5.32 \mathrm{e}-04$ & 2.03 & $1.32 \mathrm{e}-04$ & 2.01 & $3.30 \mathrm{e}-05$ & 2.00 & $8.26 \mathrm{e}-06$ & 2.00 \\
1.5 & $2.16 \mathrm{e}-03$ & 2.11 & $5.30 \mathrm{e}-04$ & 2.03 & $1.32 \mathrm{e}-04$ & 2.01 & $3.30 \mathrm{e}-05$ & 2.00 & $8.23 \mathrm{e}-06$ & 2.00 \\
1.2 & $2.16 \mathrm{e}-03$ & 2.10 & $5.29 \mathrm{e}-04$ & 2.03 & $1.32 \mathrm{e}-04$ & 2.01 & $3.28 \mathrm{e}-05$ & 2.00 & $8.21 \mathrm{e}-06$ & 2.00 \\
1.1 & $2.16 \mathrm{e}-03$ & 2.11 & $5.28 \mathrm{e}-04$ & 2.03 & $1.31 \mathrm{e}-04$ & 2.01 & $3.28 \mathrm{e}-05$ & 2.00 & $8.20 \mathrm{e}-06$ & 2.00 \\
\hline \hline
\end{tabular}


Table 4: $\left\|u-u_{h}\right\|_{0, \Omega}$ for problem (4.2) with $k=2$.

\begin{tabular}{c|cccccccc}
\hline \hline$N$ & \multicolumn{2}{|c}{8} & \multicolumn{2}{c}{16} & \multicolumn{2}{c}{32} & \multicolumn{2}{c}{64} \\
\hline$\alpha$ & error & order & error & order & error & order & error & order \\
\hline 1.9 & $3.73 \mathrm{e}-05$ & 3.00 & $4.66 \mathrm{e}-06$ & 3.00 & $5.82 \mathrm{e}-07$ & 3.00 & $7.28 \mathrm{e}-08$ & 3.00 \\
1.7 & $3.73 \mathrm{e}-05$ & 3.00 & $4.66 \mathrm{e}-06$ & 3.00 & $5.83 \mathrm{e}-07$ & 3.00 & $7.32 \mathrm{e}-08$ & 2.99 \\
1.5 & $3.73 \mathrm{e}-05$ & 3.00 & $4.67 \mathrm{e}-06$ & 3.00 & $5.84 \mathrm{e}-07$ & 3.00 & $7.38 \mathrm{e}-08$ & 2.99 \\
1.2 & $3.73 \mathrm{e}-05$ & 3.00 & $4.67 \mathrm{e}-06$ & 3.00 & $5.87 \mathrm{e}-07$ & 3.00 & $7.50 \mathrm{e}-08$ & 2.97 \\
1.1 & $3.73 \mathrm{e}-05$ & 3.00 & $4.67 \mathrm{e}-06$ & 3.00 & $5.88 \mathrm{e}-07$ & 2.99 & $7.54 \mathrm{e}-08$ & 2.97 \\
\hline \hline
\end{tabular}

Table 5: $\left\|u-u_{h}\right\|_{0, \Omega}$ for problem (4.2) with $k=3$.

\begin{tabular}{c|cccccc}
\hline \hline$N$ & \multicolumn{2}{|c}{4} & \multicolumn{2}{c}{8} & \multicolumn{2}{c}{16} \\
\hline$\alpha$ & error & order & error & order & error & order \\
\hline 1.9 & $8.02 \mathrm{e}-15$ & - & $1.12 \mathrm{e}-13$ & - & $5.36 \mathrm{e}-13$ & - \\
1.7 & $3.51 \mathrm{e}-14$ & - & $2.52 \mathrm{e}-13$ & - & $1.41 \mathrm{e}-12$ & - \\
1.5 & $1.43 \mathrm{e}-13$ & - & $6.06 \mathrm{e}-13$ & - & $4.67 \mathrm{e}-12$ & - \\
1.2 & $6.30 \mathrm{e}-14$ & - & $2.59 \mathrm{e}-13$ & - & $9.09 \mathrm{e}-13$ & - \\
1.1 & $3.94 \mathrm{e}-14$ & - & $3.46 \mathrm{e}-13$ & - & $1.48 \mathrm{e}-12$ & - \\
\hline \hline
\end{tabular}

